\title{
Conservative Management of Large Traumatic Tracheal Laceration
}

Jose Sanchez-Perez ${ }^{1}$, Gabriel Rivera-Rivera ${ }^{1}$, Mario Gonzalez ${ }^{2}$, Shayanne Lajud ${ }^{3}$, Mario Corona-Ruiz ${ }^{1}$, and Antonio Riera-March ${ }^{1}$

${ }^{1}$ University of Puerto Rico School of Medicine

${ }^{2}$ University of Puerto Rico Medical Sciences Campus

${ }^{3}$ University of Puerto Rico Rio Piedras

May 2, 2021

\section{Abstract}

Tracheobronchial injuries following blunt trauma have a low incidence due to the high mortality rate before reaching hospitals. This case report advocates for conservative management in a hemodynamic patient with a large longitudinal tracheal laceration with no signs of acute complication.

Jose R. Sanchez-Perez ${ }^{1}$, Gabriel Rivera Rivera ${ }^{1}$, Mario Gonzalez-Carbonell ${ }^{1}$, Shayanne Lajud-Guerrero ${ }^{1}$, Mario Corona-Ruiz ${ }^{1}$, Antonio Riera March ${ }^{1}$

${ }^{1}$ Department of Otolaryngology Head and Neck Surgery, University Hospital of Puerto Rico, Medical Sciences Campus, San Juan, Puerto Rico.

Author Note

This article was reviewed and considered exempt of IRB review and approval.

The author declares that there is no conflict of interest or funding. All co-authors have seen and agreed with the contents of the manuscript and there is no financial interest to report. We certify that the submission is original work and is not under review at any other publication.

Correspondence concerning this article should be addressed to Jose R. Sanchez-Perez, Department of Otolaryngology Head and Neck Surgery, University of Puerto Rico School of Medicine, Medical Sciences Campus, PO Box 365067, San Juan, PR 00936-5067, Tel: 787-758-2525 Ext: 1910, E-mail: jose.sanchez34@upr.edu

Presented as a Poster at the Combined Otolaryngology Spring Meeting, virtual meeting, United States of America, April 7-11, 2021.

\section{Conservative Management of Large Traumatic Tracheal Laceration}

\section{Authorship}

Jose R. Sanchez-Perez MD ${ }^{1}$, Gabriel Rivera Rivera MD ${ }^{1}$, Mario Gonzalez-Carbonell MD ${ }^{1}$, Shayanne LajudGuerrero $\mathrm{MD}^{1}$, Mario Corona-Ruiz $\mathrm{MD}^{1}$, Antonio Riera March $\mathrm{MD}^{1}$

\section{Affiliation}

${ }^{1}$ Department of Otolaryngology Head and Neck Surgery, University Hospital of Puerto Rico, Medical Sciences Campus, San Juan, Puerto Rico.

\section{Acknowledgement}

None. 


\section{Conflict of Interest}

The author declares that there is no conflict of interest or funding. All co-authors have seen and agreed with the contents of the manuscript and there is no financial interest to report. We certify that the submission is original work and is not under review at any other publication.

\section{Keywords}

tracheobronchial injury, management of tracheobronchial injuries, tracheal laceration

\section{Introduction}

Tracheobronchial injury (TBI) is defined as an injury localized between the cricoid cartilage and right/left mainstem bronchus. TBI commonly results from iatrogenic or traumatic injury. Iatrogenic TBI is commonly associated with longitudinal lacerations located in the cervical trachea after a difficult intubation or tracheostomy. Conversely, TBI resulting from trauma may occur from penetrating or blunt injuries. Penetrating injuries are often due to gunshot or stabbing wounds and are commonly located at the cervical trachea. Instead, blunt trauma often occurs following a motor vehicle accident with an incidence as low as $0.4 \%-1.5 \%$. (1)

While management of iatrogenic TBI has been described in the literature, there is scant information regarding traumatic TBI specifically following blunt trauma. The objective of this article is to discuss a case report of a patient who suffered a large traumatic TBI following a motor vehicle accident and the decision to undertake conservative management rather than surgical.

\section{Case Report}

A 15-year-old male arrived at the ER following a MVA as a restrained backseat passenger. Upon initial evaluation, the patient's only complaint was chest pain with no symptoms of airway distress or voice change. Examination revealed a spontaneously breathing patient with stable vital signs and adequate oxygen saturation. Physical exam was remarkable for subcutaneous emphysema, and an upper chest seatbelt bruise (FIGURE 1 ). A Neck and Chest CT scan revealed a suspected posterior longitudinal tracheal tear along the right posterior wall with associated bilateral pneumothorax and pneumomediastinum.(FIGURE 2A and 2B) Bedside flexible bronchoscopy revealed a longitudinal through-and-through distal right posterior tracheal tear at the membranous wall measuring $4.0 \mathrm{~cm}$ and expansion upon dynamic inspiration (VIDEO CLIP 1 ). Esophageal soft tissue herniation was visualized upon inspiration without evidence of connection (VIDEO CLIP 1 ). The distal portion of the tear was approximately $0.4 \mathrm{~cm}$ from the carina. A multidisciplinary decision was made to manage the tracheal laceration conservatively without fiberoptic endotracheal intubation due to patient's stable condition. Yet, pneumothorax was managed with bilateral chest tube placement. In order to completely rule out esophageal perforation a swallow study was recommended. Reevaluation was performed at one week and the patient had resolved chest pain and subcutaneous emphysema. Bronchoscopy revealed posterior membranous tracheal laceration healed by secondary intention without evidence of granulation tissue or collapse of the lumen. Revaluation at two months revealed an intact posterior tracheal wall without granulation tissue or stenosis.

\section{Discussion}

TBI following blunt trauma is rare due to the high percentage of patients dying before reaching the hospital. (1) Due to its low incidence and high mortality rates, a high degree of suspicion and prompt airway management is essential. TBI should be suspected in any patient following blunt trauma that presents with respiratory distress, dyspnea, hemoptysis, SOB, and clinical signs involving subcutaneous emphysema, pneumomediastinum, and pneumothorax.

Evaluation for all patients concerning TBI should include flexible bronchoscopy as it helps identify the location and characteristics of the injury. In unstable patients, bronchoscopy-guided endotracheal intubation is preferred over blind intubation to avoid further airway injury. 
In hemodynamically stable patients CT imaging provides an important role in identifying TBI and concomitant injuries in the neck or chest. Scaglione et al performed CT imaging in all non-complicated patients with suspected TBI and demonstrated $94 \%$ accuracy in localizing tracheobronchial injury. The sole remaining patient showed adjacent air leaks which have been demonstrated in other studies to be associated with TBI and were confirmed via bronchoscopy. ${ }^{(1)}$ Historically TBI has been primarily managed with surgical correction regardless of etiology. Recently conservative approaches have been advocated for patients with iatrogenic injuries with certain clinical and morphological properties.

There is insufficient information regarding conservative management in patients following blunt trauma due to its low incidence. However, Gomez-Caro et al established strict criteria where conservative management can be employed in patients regardless of the mechanism of trauma, location, or length. The clinical criteria consist of hemodynamically stable patients without worsening subcutaneous or mediastinal emphysema, no open tracheal injury, or signs of sepsis related to an injury. (2) Meanwhile, Caretta et al establishes criteria for conservative management based on bronchoscopy findings. These are non-complex linear injuries involving only partial thickness of the tracheal wall. (3) Finally, Cardillo et al propose a morphological classification based on the depth of injury and states, that in an uncomplicated iatrogenic TBI, patient depth not length should direct treatment. Cardillo's study advocated that only patients with evidence of esophageal or mediastinal injury (level IIIB) should undergo primary surgery. ${ }^{(4)}$

In our case, bronchoscopy revealed an uncommon longitudinal posterior right membranous tracheal wall laceration measuring $4.0 \mathrm{~cm}$ with minimal nonperforated esophageal herniation. A decision to undergo conservative management was supported by the patients age, clinical aspect and flexible endoscopy evaluation. There is not enough evidence regarding management of TBI following blunt trauma regarding length, depth, and location. Our case report imparts another successful conservative management focusing on clinical and bronchoscopy presentation.

\section{Conclusion}

TBI has historically been managed with primary surgical correction. While conservative management has risen in patients following iatrogenic injuries there is minimal information regarding management for blunt injuries. Our case report demonstrates that conservative management proved to be safe and effective for a large linear non-complex tracheal laceration with advanced depth classification in a hemodynamically stable patient without signs of acute complication.

\section{References}

1. Scaglione M, Romano S, Pinto A, et al. Acute tracheobronchial injuries: impact of imaging on diagnosis and management implications. Eur J Radiol 2006; 59:336-43.

2. Gomez-Caro A, Ausin P, Moradiellos FJ, et al. Role of conservative medical management in tracheobronchial injuries. J Trauma 2006; 61: 1426-34

3. Carretta A., Melloni G, et al. Conservative and Surgical treatment of Acute Posttraumatic Tracheobronchial injuries. World J Surg 2011; 35: 2568-2574

4. Cardillo G, Carbone L, et al. Tracheal laceration after endotracheal intubation: a proposed morphological classification to guide non-surgical treatment. Eur J Cardiothoracic Surgery 2010; 37: 581-587

\section{Figures:}

Figure 1 demonstrates the presence of diagonal bruising also known as seatbelt sign across the patient's chest.

Figure 2 is a Neck \& Chest CT with IV contrast demonstrating a Sagittal view on figure 2A and an Axial view on figure 2B of a longitudinal right posterior tracheal wall defect at level of T1-T2. 


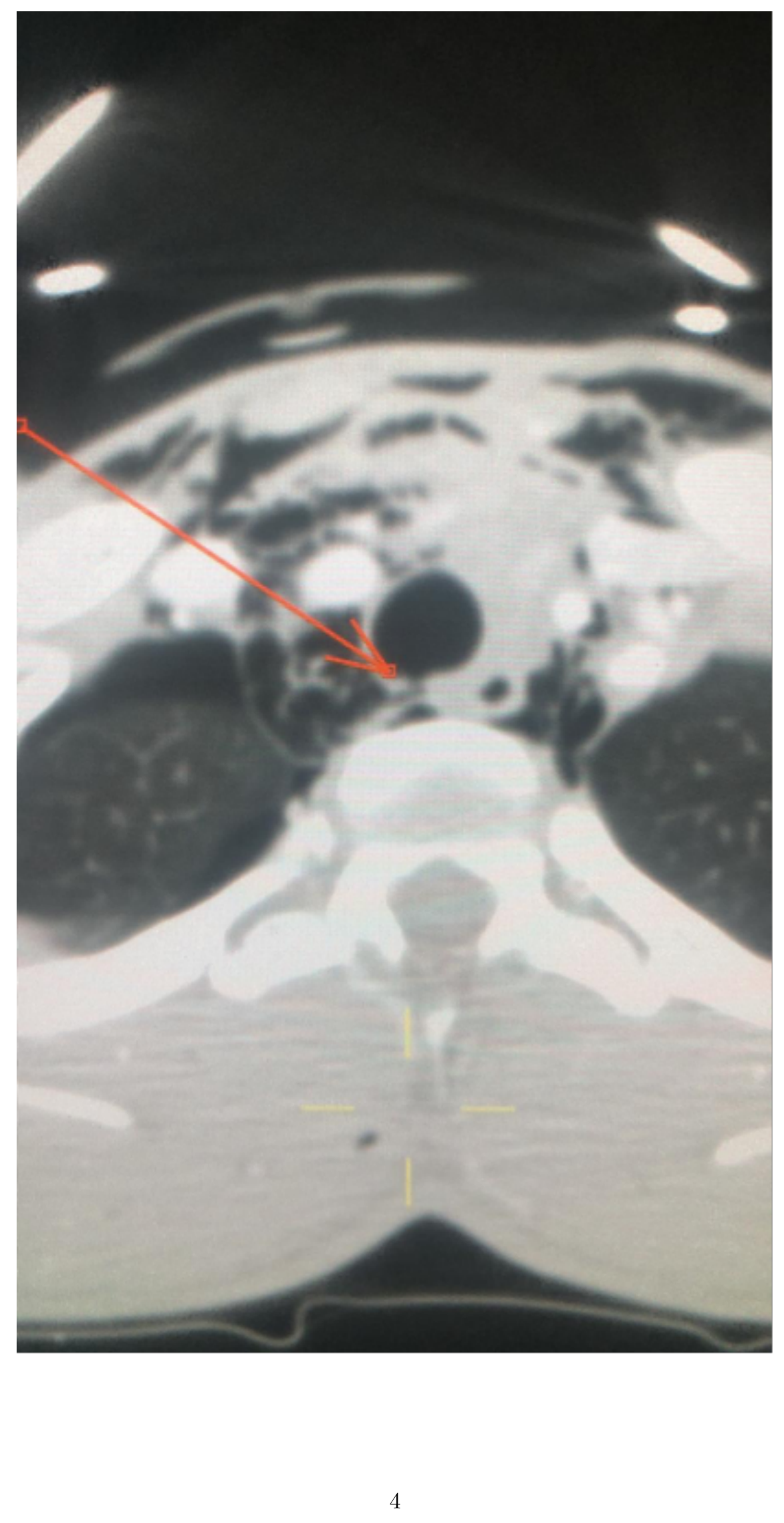




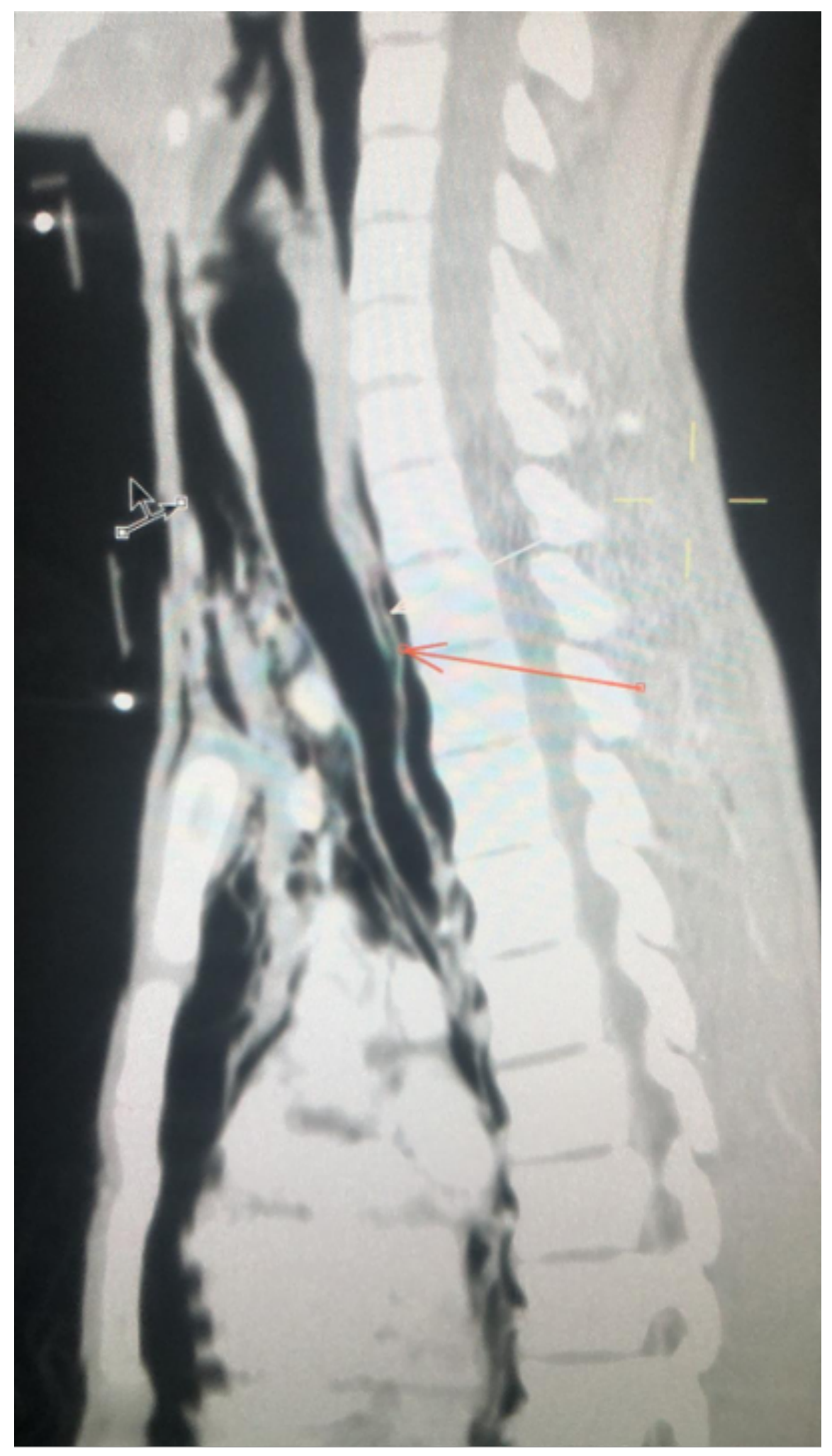




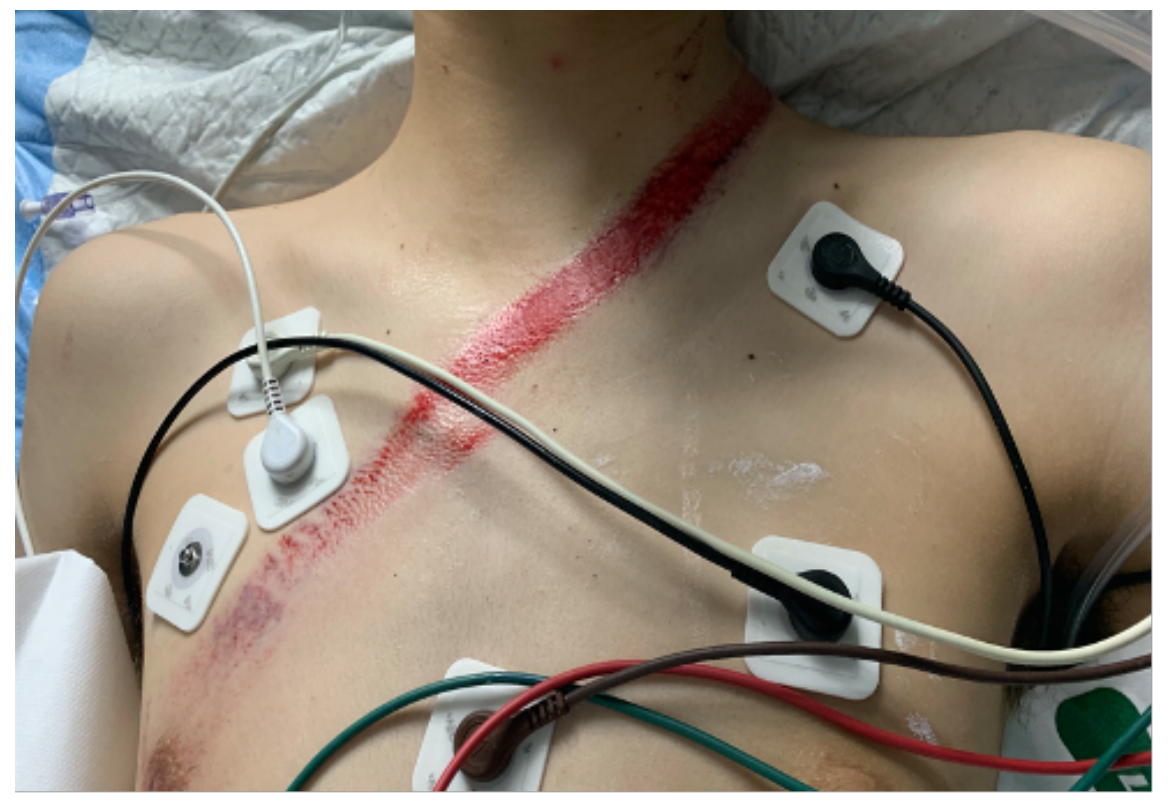

